\title{
A Variable Stiffness Soft Gripper Using Granular Jamming and Biologically Inspired Pneumatic Muscles
}

Loai A. T. Al Abeach, Samia Nefti-Meziani, T. Theodoridis, Steve Davis

Autonomous Systems and Robotics Research Centre, University of Salford, Salford M5 4WT, UK (e-mail:1.a.t.alabeach@edu.salford.ac.uk)

\begin{abstract}
As the domains in which robots operate change the objects a robot may be required to grasp and manipulate are likely to vary significantly and often. Furthermore there is increasing likelihood that in the future robots will work collaboratively alongside people. There has therefore been interest in the development of biologically inspired robot designs which take inspiration from nature. This paper presents the design and testing of a variable stiffness, three fingered soft gripper which uses pneumatic muscles to actuate the fingers and granular jamming to vary their stiffness. This gripper is able to adjust its stiffness depending upon how fragile/deformable the object being grasped is. It is also lightweight and low inertia making it better suited to operation near people. Each finger is formed from a cylindrical rubber bladder filled with a granular material. It is shown how decreasing the pressure inside the finger increases the jamming effect and raises finger stiffness. The paper shows experimentally how the finger stiffness can be increased from 21 to $71 \mathrm{~N} / \mathrm{m}$. The paper also describes the kinematics of the fingers and demonstrates how they can be position-controlled at a range of different stiffness values.
\end{abstract}

\section{Keywords}

Biologically inspired robots, variable stiffness actuation, soft robotics, soft grippers, pneumatic muscles

\section{Introduction}

Traditional robot grippers tend to be inflexible meaning they are only suited to grasping a small range of products. This is not a problem when a robot is always handling the same object but creates a problem if the robot is required to grasp many very different items. Each item may require its own gripper and this can make the use of a robot prohibitively complex and expensive. This has led to the development of multi-functional dexterous robot hands such as the Utah/MIT hand [1], Anthrobot Hand [2], Robonaut Hand [3], DLR-Hands [4] and the Metamorphic Hand [5], to name just a few, which are inspired by the human hand. However, despite being able to replicate human like motions and grasps these hands have typically used stiff actuators which behave in a very different manner to organic muscle, meaning all the abilities of the human hand are not replicated. 


\section{A Variable Stiffness Soft Gripper Using Granular Jamming and Biologically Inspired Pneumatic Muscles}

Organic muscle is unique in its ability to provide power for creatures which vary in size from insects up to the largest creatures found in the sea. It is also able to operate in a broad range of climatic conditions. Organic muscle also provides the ability to modulate both stiffness and position, which means an organic systems can switch between being highly compliant or much stiffer depending upon the task to be undertaken. This ability is of potential interest to roboticists as it would allow robots to be compliant when safe human interaction is required but stiffer when the task demands it. Unfortunately organic muscle is not an engineering technology and so not suited to machine operation. There have been many attempts to develop new actuators which superficially replicate the performance of organic muscle. One of the more promising are pneumatic muscles, developed in the 1950s [6,7] which attempt to emulate the 'soft' compliant structure of muscle and combine this with the power, robustness, accuracy, and endurance of mechanical drives. Pneumatic muscles have a range of characteristics which make them of interest to robotics researchers [8]:

- $\quad$ They are available in any length and diameter with larger diameter muscles producing higher contractile force.

- $\quad$ They have exceptionally high power and force to weight/volume ratios.

- $\quad$ Displacement (contraction) is typically $30-35 \%$ of the dilated length and this is comparable with natural muscle.

- $\quad$ They are highly flexible, soft in contact and have excellent safety potential.

- Contractile force to cross-sectional area can be over $300 \mathrm{~N} / \mathrm{cm}^{2}$ compared to $20-40 \mathrm{~N} / \mathrm{cm}^{2}$ for natural muscle.

- $\quad$ They can operate safely in many environments and are safe in explosive/gaseous states.

There have been examples of multi fingered dexterous grippers that are actuated by pneumatic muscles in the past, for example Davis et al [9] and the Shadow Hand [10]. These dexterous hands have demonstrated impressive functionalities and replicate the human hand closely. However, the human hand should not be considered the perfect gripper and exact replication of the human hand may not be the best approach to developing a multi-purpose dexterous gripper. Dexterous grippers based on the human hand have limitations, they tend to grasp objects using a relatively low number of point contacts. This means there are areas of localised force on the object being grasped which can lead to damage if the object is fragile. Also in the future robots are likely to work collaboratively with people and it is essential that this interaction be safe. These dexterous robot hands also tend to have high masses and associated inertias which means they are not suited to close human interaction [11].

The relatively new field of soft robotics has the potential to address the problems of safe human interaction and the ability to handle delicate objects. Researcher in the field of soft robotics take inspiration from biology, particularly animals which do not have rigid skeletons, and attempt to develop robots without rigid links [12]. Examples include robots based on caterpillars [13], the octopus [14] and worms [15] among others. 


\section{A Variable Stiffness Soft Gripper Using Granular Jamming and Biologically Inspired Pneumatic Muscles}

Soft robots tend to be lightweight and have low inertia, they are also highly compliant and can conform to surfaces they come into contact with. This ability can help to address the two issues highlighted above. In terms of safety it means in a collision with a person contact stresses are distributed over a larger area, minimising localised forces and lowering the chance of injury [12]. The ability to distribute grasp forces over a larger area can also reduce the chance of damage as localised forces are again reduced. The larger contact area can also result in a more secure grasp.

There have been a number of these biologically inspired soft robot grippers developed including tendon driven grippers [16], [17], tendon and pressure actuation for a bio-inspired manipulator based on an antagonistic principle [18] and fluid powered grippers [19]-[22]. In most cases these grippers are unable to change their stiffness and whilst highly compliant fingers may be desirable for grasping some products, others (e.g. highly deformable products) may require a stiffer gripper. One approach to achieving this was presented by Al Abeach [23] who developed a multi fingered dexterous gripper which used both extending and contracting pneumatic muscles to allow the fingers to vary their stiffness. Shiva introduce another approach where the stiffness control is achieved using pneumatic and tendon actuation operating antagonistically [24].

This paper explores the use of pneumatic muscles to actuate a soft dexterous gripper and granular jamming to vary its stiffness. Granular jamming exploits the fact that in a fixed volume container, granules of material can move easily relative to each other. However, if a pressure is applied to the granules which forces them tightly against each other they become much more difficult to move.

Granular jamming techniques have previously been used in the development of grippers and variable stiffness robots. Jiang proposed a high DOF variable stiffness joint, and demonstrated its use in a miniature snake-like robot [25]. The variable stiffness ability was achieved by using granular jamming, they applied a vacuum to a granular-filled membrane which resulted in increased stiffness due to the jamming of the granules. They demonstrated a stiffness increase of $400 \%$. Cheng proposed a continuum soft robot arm based on the use of the granular jamming techniques to construct the arm [26]. Tension cables and spooling motors were used to control the bending of the continuum arm and granular jamming allowed the stiffness to be tuned.

Brown proposed a universal gripper constructed from a granular material placed in single nonporous elastic bag instead of using individual fingers [27]. The gripper achieves gripping by deforming around the object to be held before granular jamming is used to solidify it using a reduction in air pressure. A $0.5 \%$ reduction in volume of the gripper was found to be associated with the jamming allowing the gripper to grasp the object that it had enveloped. The gripper was able to successfully and securely grasp a range of objects whose shape was not known a priori. Other jamming techniques have been developed which exploit layer jamming [28]. These systems consist of concentric layers of material which when forced against each other increase the level of friction in the system and this results in increased stiffness. 


\section{A Variable Stiffness Soft Gripper Using Granular Jamming and Biologically Inspired Pneumatic Muscles}

This paper first details the concept of using granular jamming to create a variable stiffness finger. The amount by which the stiffness of the finger can be varied is then determined experimentally. The kinematics of the gripper are then briefly discussed before the paper describes the design of a three fingered variable stiffness soft gripper which uses soft pneumatic muscles to control flexion of the fingers. The ability to accurately control the position of the fingers at a range of stiffness values is then explored experimentally before some simple grasps are demonstrated.

\section{Granular Jamming Finger Design}

The method to be used to vary the stiffness of the proposed end effector is granular jamming. A single granular jamming continuum actuator based finger was constructed to investigate the variable stiffness capabilities experimentally. Figure 1 shows the materials used to construct the granular jamming continuum finger.

The rubber bladder in figure 1 is $10 \mathrm{~mm}$ in diameter and $160 \mathrm{~mm}$ in length, it is used to contain all the granular material and is nonporous. The granular material is then inserted into the rubber tube to completely fill it, the granular material chosen to be used in this prototype is rice, due to its relatively small size compared to the diameter of the bladder. Furthermore rice was previously used by the authors to investigate varying the stiffness of pneumatic muscles [29]. An investigation of different granular materials can be found in the work of Jiang et al [30]. Two 3D printed plugs were inserted into the open ends of the bladder, and secured in place, to seal it. One of the plugs contained a hole which would allow air to be passed into and out of the bladder. The granular jamming effect requires that a vacuum be applied to the bladder to reduce the air pressure in it, however, that would have the effect of sucking the granular material out of the finger. For this reason a foam plug was used in the air inlet to prevent granular materials leaking out during operation of the vacuum valve. A woven nylon shell was then added to form an out layer of the granular jamming continuum finger. This braided shell is not needed for successful operation of the granular jamming finger but it was included for two reasons, firstly it provides some limited protection to the rubber bladder, reducing the chance of punctures, but it also allowed the attachment and correct routing of the tendons which are used to flex the finger, this will be discussed in further detail in later sections.

The system is compliant because the granular materials are able to move/flow. This means when an external force is applied to the finger its position will change and the granules inside will become rearranged. At atmospheric pressure this is relatively easy to achieve as the only thing hindering motion of the granules is the friction between adjacent grains. However, when vacuum is applied, the grains are pressed together and this results in an increase in the frictional forces between the grains and this makes it harder for them to move, as can be seen in figure 2. The fact that a greater force is needed to deform the finger means that it has become a stiffer structure. 


\section{A Variable Stiffness Soft Gripper Using Granular Jamming and Biologically Inspired Pneumatic Muscles}

The system is superficially inspired by a muscular hydrostat such as the human tongue or an elephant's trunk. Although the system does not include a fluid, the granules are able to flow in a manner analogous to that of a fluid. At greater jamming pressures the granules flow less easily and the system appears stiffer.

\section{Granular Jamming Finger Stiffness Characteristics}

The test rig used to analyse the bending stiffness of the fingers can be seen in figure 3 . The granular jamming continuum finger was positioned so as to protrude vertically upwards. A tendon cable was then attached to the fingertip which passed over a pulley to a vertically suspended load. As the load is increased it increases the force on the tendon and as a result the horizontal force applied to the fingertip is increased. This resulted in a lateral displacement of the fingertip which could be measured from the unloaded position using Vernier callipers. From this measured displacement and the known force being applied via the tendon the lateral finger bending stiffness could be calculated. The test rig also consisted of a venturi based vacuum generator and a pressure gauge which allowed the negative pressure in the finger to be controlled manually. This allowed the stiffness experiments to be performed at a range of granular jamming pressures. The height of the aluminium pulley could be adjusted to ensure that the tendon was always horizontal and so force was consistently applied to the fingertip in a lateral direction. If the height of the pulley was not changed as the finger flexed the tendon would not remain horizontal and therefore only a component of the load force would be applied laterally to the fingertip. The pulley's height was adjusted each time a new load was applied to ensure the force was always applied in the correct direction. Despite this approach, it is not possible to have the tendon angle equal to exactly 90 degrees, for this reason all experiments were repeated five times and an average value taken. This approach minimises the effects of the angular error in the final results.

The experimental procedure began by removing any load and then venting the granular jamming continuum finger to atmosphere to ensure that the grains were in their least jammed configuration, allowing them to move as freely as possible. The load was then increased in $0.25 \mathrm{~N}$ increments from zero up to a maximum of $1.5 \mathrm{~N}$. After each load was applied the height of the pulley was manually adjusted to ensure the force $\mathrm{F}_{\mathrm{LOAD}}$ was applied laterally to the fingertip. This force caused the finger to bend, as can be seen in the figure, and the lateral displacement of the fingertip was then measured and recorded. The load was then reduced in $0.25 \mathrm{~N}$ increments back down to zero and the displacement at each load was again recorded. This procedure was repeated five times and an average displacement value was calculated. The experiment was repeated at reducing jamming pressures, down to $-80 \mathrm{kPa}$ in decrements of $-20 \mathrm{kPa}$. The displacement of the finger as force is applied for each of the five pressure values can be seen in figure 4. It can be seen that at lower pressures, i.e. greater jamming pressures the finger displaces less that at higher pressures when the same load is applied. 


\section{A Variable Stiffness Soft Gripper Using Granular Jamming and Biologically Inspired Pneumatic Muscles}

It can be seen that the relationship between displacement and force is approximately linear. It is therefore possible to add linear lines of best fit to each of the five sets of data. The bending stiffness for the granular jamming continuum finger can then be calculated by taking the gradient of each linear approximation. Figure 5 shows the calculated bending stiffness of the granular jamming continuum finger as the pressure inside the finger is reduced.

From the figure 5 it can be seen that reducing the pressure and therefore increasing the amount of jamming force has a significant impact on the stiffness, increasing it from 21 to $71 \mathrm{~N} / \mathrm{m}$ as the pressure is reduced from atmospheric pressure to $-80 \mathrm{kPa}$. It can also be seen that a relatively small pressure change has a much more significant impact on the stiffness at lower pressures than it does at higher one. For example reducing the pressure from 0 to $-20 \mathrm{kPa}$ results in a stiffness increase of just $2 \mathrm{~N} / \mathrm{m}$, however, reducing it from $-60 \mathrm{kPa}$ to $-80 \mathrm{kPa}$ (the same $20 \mathrm{kPa}$ reduction) results in a stiffness increase of $33 \mathrm{~N} / \mathrm{m}$.

Figure 6 shows the percentage increase in the granular jamming finger bending stiffness as the pressure in it is decreased. It can be seen that decreasing the jamming pressure to $-80 \mathrm{kPa}$ results in an increase in stiffness of $235 \%$. Again it is clear that the relationship is highly nonlinear. A $20 \mathrm{kPa}$ reduction in pressure from a $0 \mathrm{kPa}$ staring pressure increases stiffness by $9 \%$ where as a $20 \mathrm{kPa}$ reduction from a starting pressure of $-60 \mathrm{kPa}$ results in a substantially higher increase of $155 \%$.

This section has proven that the proposed granule-filled finger is able to significantly vary its stiffness through granular jamming. However, the finger is unactuated, the next section will discuss the kinematics of the finger and show how through the use of tendons the finger can be made to flex.

\section{Finger Kinematics}

In the proposed gripper each finger will be a continuum link with the body of the link being formed from the granular jamming bladder. Tendons are then used to transmit actuation forces to the finger. Three tendons are used to apply force to the finger, these are equally space $\left(120^{\circ}\right.$ apart when viewed from the end of the finger) around the circumference of the finger. Three tendons are used as this is the minimum number required to allow the finger to bend in all directions. The three tendons are secured to the tip of the finger and then run along the length of the finger to the actuators. As each tendon is pulled it will cause the finger to flex in one of three directions. If multiple tendons are displaced the finger will move in multiple direction simultaneously this means that by an appropriate combination of tendon motions the finger can be made to move in all directions.

In order to control the position of the end of the finger it is vital that a kinematic analysis is performed which will relate motion of the tendons to the magnitude and direction of finger bending. The analysis assumes that the finger has a constant circular cross-section, the tendon remains in contact with the sides of the finger at all times (the way this is achieved will be discussed in 
the next section) and that as the finger bends it forms a perfect arc. Figure 7 shows a schematic of the proposed actuated finger which has three equally space tendons around its circumference. The lengths of tendon 1, tendon 2 and tendon 3 that travel along each side of the finger is given by $L_{1}, L_{2}$ and $L_{3}$ respectively. The overall length of the finger measured from the centre of the finger base to the centre of its tip is defined by L. The radius of the finger (i.e. the distance of the tendons from the central axis of the finger) is represented by $\mathrm{r}_{\mathrm{o}}$.

The kinematic analysis developed here is based on the approach presented by Godage et al [31]. Four properties are used to describe the position of the free end of the finger relative to the base. The length of the finger, which will remain constant, the radius of curvature of the finger is defined as $\lambda, \emptyset$ is the difference in angle between the base and end of the finger and $\theta$ is the angle in which the finger points relative to the base coordinate frame.

The kinematics of finger are described using the following four equations presented by Godage et al [31]:

$$
\begin{gathered}
\lambda=\frac{\left(L_{1}+L_{2}+L_{3}\right) r_{o}}{2 \sqrt{L_{1}{ }^{2}+L_{2}{ }^{2}+L_{3}{ }^{2}-L_{1} L_{2}-L_{1} L_{3}-L_{2} L_{3}}} \\
\emptyset=\frac{2{\sqrt{L_{1}{ }^{2}+L_{2}{ }^{2}+L_{3}{ }^{2}-L_{1} L_{2}-L_{1} L_{3}-L_{2} L_{3}}}_{3 r_{o}}}{}
\end{gathered}
$$$$
\theta=\tan ^{-1}\left(\frac{\sqrt{3}\left(L_{3}-L_{2}\right)}{L_{2}+L_{3}-2 L_{1}}\right)
$$$$
L=\lambda \emptyset
$$

If equations (1), (2) and (4) are combined then an expression describing the finger length can be determined:

$$
L=\frac{L_{1}+L_{2}+L_{3}}{3}
$$

Equation (5) suggests that it is possible to reduce the length of the finger by reducing the lengths of all the contractor muscles. However, this is not possible because the grains inside the finger are incompressible and so the volume of the finger is a constant. Instead of shortening the finger would buckle if $\mathrm{L}_{1}, \mathrm{~L}_{2}$ and $\mathrm{L}_{3}$ are all reduced simultaneously. It is therefore vital that when controlling the position of the fingers equation (5) is used to ensure the finger length remains constant. That is to say if a tendon shortens, to create bending the two other tendons must increase in length to ensure L remains unchanged.

Equations (1)-(3) can be combined to give the Cartesian positions $\left(\mathrm{x}_{\mathrm{i}}, \mathrm{y}_{\mathrm{i}}, \mathrm{z}_{\mathrm{i}}\right)$ of the fingertip in the base coordinate systems as follows:

$x_{i}=(\lambda-\lambda \cos \phi) \cos \theta$ 
$y_{i}=(\lambda-\lambda \cos \phi) \sin \theta$

$z_{i}=\lambda \sin \phi$

\section{Gripper Design}

There are many actuator types that could be used to apply tension to the tendons but the actuators chosen for use in this research are soft pneumatic muscles. There are a number of reasons why this type of actuator was chosen. Firstly it is lightweight and has a high power to weight ratio [8], which keeps the weight and therefore associated inertia of the gripper low which is vital for safe operation near people. The second reason is that the actuator is compliant.

Although it is granular jamming that allows the stiffness of the finger to be adjusted, the system must use compliant actuators. Consider the case where the finger is bent as shown in figure 7. If a force is applied to the end of the finger in an attempt to straighten it, this will apply a tension to tendons 1 and 2 which will try to extend them. If the tendons are inextensible and the actuators used to displace them are stiff, $\mathrm{L}_{1}$ and $\mathrm{L}_{2}$ will remain fixed and the tip of the finger will not move. This will mean that despite the variable stiffness abilities of the finger the actuators actually mean the gripper is stiff. If compliant actuators are used however, when the force is applied to the fingertip the actuators will displace elastically, allowing the tendons to move and the finger to be repositioned (i.e. it is compliant).

The conceptual design of a single finger for the gripper is shown in figure 8 . The finger is formed from a granular jamming link as descried in the previous sections. The finger has a diameter of $15 \mathrm{~mm}$ and a length of $160 \mathrm{~mm}$. Three equally spaced nylon tendons are attached to the end of the finger and pass along its sides and through a fixed position plate at the base of the finger. To ensure the tendons remain in contact with the sides of the finger at all times they pass through a series of tendon loops. These loops are positioned at regular intervals along the length of the finger. These are formed from nylon cable ties which are fed through adjacent openings in the braided shell placed on the outside of the finger.

The finger is attached to a forearm like structure which contains all the pneumatic muscles used to flex and extend the finger. Each muscle has a maximum unpressurised length of $240 \mathrm{~mm}$ and a contracted length at $400 \mathrm{kPa}$ of $180 \mathrm{~mm}$ and each muscles has a maximum force output $\mathrm{F}_{\mathrm{C}}$ of approximately $200 \mathrm{~N}$ at $400 \mathrm{kPa}$ (determined experimentally).

The complete gripper consists of three fingers and so the forearm contains nine pneumatic muscles arrange in three groups of three, with each group providing power to one finger.

The complete gripper can be seen in Figure 9, as can be seen, linear potentiometers are located between the pneumatic muscles and the fingers. Each tendon is attached to one potentiometer which is used to measure the contraction of the muscles and thus 
using the kinematic analysis above, the position of the finger. These sensors are vital for closed loop control of the finger positions.

Each muscle is attached to an individual MATRIX 3-3 solenoid valve allowing air to be supplied to or vented from a single muscle when required. The valves are pulse width modulated to allow the flowrate of air into and out of the muscle to be varied.

Overall control of the hand is achieved using an Arduino UNO linked to MATLAB. To allow the Arduino to generate the necessary PWM signals to drive all of the 18 pneumatic valves (9 fill and 9 vent) two Adafruit 16-channels PWM/Servo Shields were used to expand the PWM capacity of the Arduino. Amplifier circuits were also required to amplify the voltage and current output to that required by the valves.

\section{Control and Performance Evaluation of Variable Stiffness Granular Jamming Gripper}

To allow the gripper to be used practically it needs to be controllable in a closed-loop manner. A PID controller was programmed to control the displacement (length) of each of the contractor muscles. Although it is widely accepted that PID is not best suited to soft robots PID controllers have been used successfully in the past to control pneumatic muscles as their force/displacement/pressure characteristics can be approximated to linear relationships [8].

The position of each fingertip is determined by the relative length of the three pneumatic muscles used to power it. The controller reads the length of the muscle from the potentiometer and then generates the necessary PWM control signals to vary the flow of air in and out of the muscles. The PID controller was tuned using the Ziegler-Nichols method [32].

In order to assess the performance of the controller, the response to a step position change and the ability to track a sinusoidal input were tested. In the experimental procedure used in all the following tests the gripper was suspended in a test rig so that it was pointed vertically downwards as seen in figure 9. The finger was then programmed to flex and extend. This required that the kinematic equation (5) be used to ensure that as a single muscle contracted in order to flex the finger the other two muscles relaxed/elongated to ensure the finger length $(\mathrm{L})$ remained constant.

For the proposed end effector to operate in various stiffness modes, it is vital that it can be controlled irrespective of the pressure in the granular jamming continuum actuators. To determine if this was possible, step and sinusoidal response experiments were conducted over a range of different granular jamming pressures.

Figure 10 shows the system response when a step input is applied to each contractor muscle. The first contractor muscle causes flexion of the finger whilst the other two muscles cause extension. The step signal applied to the first muscle was $180^{\circ}$ out of phase to the signal applied to the second and third muscles. This caused the finger to repeatedly flex and extend. These first results were obtained with a granular jamming pressure of $0.0 \mathrm{kPa}$. 


\section{A Variable Stiffness Soft Gripper Using Granular Jamming and Biologically Inspired Pneumatic Muscles}

It can be seen that the response is broadly similar for the three muscles with each overshooting briefly before settling at the target position after approximately 0.5 seconds. It can also be seen that the response when the muscles extend from $20 \mathrm{~mm}$ back to $0 \mathrm{~mm}$ displacement is not as rapid as when moving in the other direction. The reason for this is the muscles are only capable of contracting, extension is caused by the antagonistic force from the other muscles.

The experiment was repeated with a $-40 \mathrm{kPa}$ pressure applied to the granular jamming continuum fingers, from figure 6 this represented an increase in the finger's stiffness of $34 \%$ over the previous test. Figure 11 shows the step response for the first contractor muscle (muscle causing finger flexion). It can be seen that despite the increased finger stiffness the performance of the controller is broadly the same as when the jamming pressure is zero.

Figure 12 shows the controller step response when the finger jamming pressure was at $-80 \mathrm{kPa}$; this represents a $235 \%$ increase in stiffness compared to the stiffness at a jamming pressure of $0.0 \mathrm{kPa}$. Again it can be seen that, even when employing a relatively basic form of controller, the finger responds to the step position change with a similar degree of accuracy and response time at all jamming pressures.

The following figures show the ability of the controller to track a sinusoidal input signal. The range of motion is across $18 \mathrm{~mm}$ of muscle displacement from 2 to $20 \mathrm{~mm}$ which resulted in the finger flexing and extending. This caused the fingertip to move by approximately $80 \mathrm{~mm}$. The experiment was conducted at a frequency of $0.33 \mathrm{~Hz}$. During the experiment the other two muscles were provided with target positions which were calculated using the kinematic analysis to ensure the finger length (L) remained constant. If this was not done then the finger would be compressed and likely buckle.

Figure 13 shows the ability of the controller to track a sinusoidal input signal that induced flexion and extension of the finger when no vacuum was applied to the finger (i.e. a jamming pressure of $0.0 \mathrm{kPa}$ ). It can be seen that the system tracks the input signal well.

The experiment was repeated with a jamming pressure equal to $-40 \mathrm{kPa}$, again representing an increase in finger stiffness of $34 \%$. The result are shown in figure 14 . Finally the experiment was repeated at a $-80 \mathrm{kPa}$ jamming pressure which resulted in a $235 \%$ increase in finger stiffness, these results can be seen in figure 15.

From Figures 13, 14 and 15 it can be seen that the tracking is equally good during the rising part of the cycle i.e. where the muscle is contracting. However, as the target position returns to the minimum value there is a tracking error which becomes more significant at lower jamming pressures. It is likely that this is the result of friction. The muscles cannot actively extend, instead the muscle which caused flexion relies on the force from the other two muscles to act antagonistically to straighten the 


\section{A Variable Stiffness Soft Gripper Using Granular Jamming and Biologically Inspired Pneumatic Muscles}

finger, thus applying force to the tendon which in turn extends the muscle used to provide flexion. At a jamming pressure of $0 \mathrm{kPa}$ there is relatively little friction in the finger and so as the finger extends easily and the antagonistic force is transmitted to the flexion muscle extending it. However, at $-80 \mathrm{kPa}$ the finger becomes more difficult to flex due to friction caused by the granular jamming. This friction is likely preventing the edge of the finger which was previously flexed from extending fully and so the flexion muscle is not pulled back to its initial extended length.

\section{Grasping}

The control hardware and scheme described preciously was extended to all three fingers to allow closed loop position control for the whole three-finger gripper. This was necessary to allow the gripper to be used practically to grasp objects. Figure 15 shows the gripper grasping two different objects. In figure 16(a) the gripper is grasping a ball where the contact area extends along the entire length and width of the fingers and a low finger stiffness ( $0 \mathrm{kPa}$ jamming pressure). It can be seen that as the fingers make contact with the ball they deflect around it thus maximising the contact area. Figure 16(b) shows the gripper grasping a deformable paper cup using only the fingertips. In this scenario the fingers had a high stiffness (-80kPa jamming pressure) ensuring the finger remained more rigid and the grasp consisted of three point contacts.

\section{Conclusion}

This paper has presented the design, analysis and testing of a variable stiffness, three fingered soft gripper. In low stiffness mode the fingers are highly compliant meaning they will deform around objects, this is particularly useful if the object is fragile or delicate.. However, compliant fingers are not suited to grasping all objects and at times a more rigid grasp is required. For this reason the gripper is able to vary its stiffness through the use of granular jamming.

Each of the gripper's fingers is formed from a cylindrical rubber bladder filled with a granular material. The paper has shown that decreasing the pressure inside the finger increases the jamming effect and raises the stiffness of the finger. The paper has shown experimentally that the stiffness of each finger can be increased by $235 \%$. Each finger is actuated by three pneumatic muscles which apply forces via tendons to each finger to allow it to bend in all directions. The paper introduced the basic kinematic equations to describe the position of the fingers and described how these must be used when controlling the three actuators to ensure the finger does not buckle when in operation.

The paper has demonstrated that the gripper can be controlled using a PID controller as the operation of the pneumatic muscles can be approximated to linear. However, the use of other control techniques will be part of future work. It has been seen that due to the effects of friction the position accuracy when extending the fingers (i.e. opening the grasp) reduces when the stiffness of the finger is raised. The paper has only demonstrated position control but future work will also seek to implement other types of control on the gripper such as force and slip. 
A Variable Stiffness Soft Gripper Using Granular Jamming and Biologically Inspired Pneumatic Muscles

\section{Acknowledgment}

This work was supported by the Ministry of Higher Education and Scientific Research, Iraq.

\section{References}

[1] Jacobsen S C, Wood J E, Knutti D F, Biggers K B. The UTAH/MIT dextrous hand: Work in progress. The International Journal of Robotics Research, 1984, 3, 21-50.

[2] Ali M S, Kyriakopoulos K J, Stephanou H E. The kinematics of the Anthrobot-2 dextrous hand. Proceedings of the IEEE International Conference on Robotics and Automation, Atlanta, USA, 1993, 705-710.

[3] Diftler M A, Mehling J S, Abdallah M E, Radford N A, Bridgwater L B, Sanders A M, Hargrave B. Robonaut 2-the first humanoid robot in space. Proceedings of the IEEE International Conference on Robotics and Automation, Shanghai, China, $2011,2178-2183$

[4] Grebenstein M, Albu-Schäffer A, Bahls T, Chalon M, Eiberger O, Friedl W, Höppner H. The DLR hand arm system. Proceedings of the IEEE International Conference on Robotics and Automation, Shanghai, China, 2011, 3175-3182.

[5] Gao Z, Wei G, Dai J S. Inverse Kinematics and Workspace Analysis of the Metamorphic Hand. Journal of Mechanical Engineering Science, 2014, 5, 965-975.

[6] Schulte H F. The Characteristics of the McKibben Artificial Muscle. In the Application of External Power in Prosthetics and Orthotics, 1962, 94-115.

[7] Chou C P, Hannaford B. Measurement and Modelling of McKibben Pneumatic Artificial Muscles. IEEE Transactions On Robotics and Automation, 1996, 12, 90-102.

[8] Davis S, Tsagarakis N, Canderle J, Caldwell D. Enhanced modelling and performance in braided pneumatic muscle actuators. The International Journal of Robotics Research, 2003, 22(3-4), 213-227.

[9] Davis S. Caldwell D. A Dexterous Robot Hand for Museum Exhibition. Design, Installation and Maintenance. An International Interdisciplinary Journal, 2010, 13-6, 1859-1867.

[10] Shadow Robot Company LTD (2015). Shadow Dexterous Hand. Retrieved from https://www.shadowrobot.com/products/dexterous-hand/

[11] Bicchi A, Tonietti G. Fast and" soft-arm" tactics [robot arm design]. IEEE Robotics \& Automation Magazine, 2004, 11(2), $22-33$.

[12] Kim S, Laschi C, Trimmer B. Soft robotics: a bioinspired evolution in robotics. Trends in biotechnology, 2013, 31(5), 287294.

[13] Lin H T, Leisk G G, Trimmer B. GoQBot: a caterpillar-inspired soft-bodied rolling robot. Bioinspiration \& biomimetics, 2011, 6(2), 026007. 
[14] Laschi C, Cianchetti M, Mazzolai B, Margheri L, Follador M, Dario P. Soft robot arm inspired by the octopus. Advanced Robotics, 2012, 26(7), 709-727.

[15] Seok S, Onal C D, Wood R, Rus D, Kim S. Peristaltic locomotion with antagonistic actuators in soft robotics. Proceedings of the IEEE International Conference on Robotics and Automation, Anchorage, AK, USA, 2010, 1228-1233.

[16] Hassan T, Manti M, Passetti G, d'Elia N, Cianchetti M, Laschi C. Design and development of a bio-inspired, under-actuated soft gripper. Proceedings of the IEEE International Conference on Engineering in Medicine and Biology Society (EMBC), Milan, Italy, 2015, 3619-3622.

[17] Rateni G, Cianchetti M, Ciuti G, Menciassi A, Laschi C. Design and development of a soft robotic gripper for manipulation in minimally invasive surgery: a proof of concept. Meccanica, 2015, 50(11), 2855-2863.

[18] Maghooa F, Stilli A, Noh Y, Althoefer K, Wurdemann H A. Tendon and pressure actuation for a bio-inspired manipulator based on an antagonistic principle. Proceedings of the IEEE International Conference on Robotics and Automation, Seattle, WA, USA, 2015, 2556-2561.

[19] Katzschmann R K, Marchese A D, Rus D. Autonomous object manipulation using a soft planar grasping manipulator. Soft Robotics, 2015, 2(4), 155-164.

[20] Mosadegh B, Polygerinos P, Keplinger C, Wennstedt S, Shepherd R F, Gupta U, Shim J, Bertoldi K, Walsh C J, Whitesides G M. Pneumatic networks for soft robotics that actuate rapidly. Advanced Functional Materials, 2014, 24(15), $2163-2170$.

[21] Homberg B S, Katzschmann R K, Dogar M R, Rus D. Haptic identification of objects using a modular soft robotic gripper. Proceedings of the IEEE International Conference on Intelligent Robots and Systems. Hamburg, Germany, 2015, $1698-1705$.

[22] Wakimoto S, Ogura K, Suzumori K, Nishioka Y. Miniature soft hand with curling rubber pneumatic actuators. Proceedings of the IEEE International Conference on Robotics and Automation, Kobe, Japan, 2009, 556-561.

[23] Al Abeach L A, Nefti-Meziani S, Davis S. Design of a Variable Stiffness Soft Dexterous Gripper. Soft Robotics, 2017, 4(3), 274-284.

[24] Shiva A, Stilli A, Noh Y, Faragasso A, De Falco I, Gerboni G, Wurdemann H A. Tendon-based stiffening for a pneumatically actuated soft manipulator. IEEE Robotics and Automation Letters, 2016, 1(2), 632-637.

[25] Jiang A, Ataollahi A, Althoefer K, Dasgupta P, Nanayakkara T. A variable stiffness joint by granular jamming. Proceedings of the ASME 2012 international design engineering technical conferences and computers and information in engineering conference, Chicago, Illinois, USA, 2012, 267-275.

[26] Cheng N G, Lobovsky M B, Keating S J, Setapen A M, Gero K I, Hosoi A E, Iagnemma K D. Design and analysis of a robust, low-cost, highly articulated manipulator enabled by jamming of granular media. Proceedings of the IEEE International Conference on Robotics and Automation, Saint Paul, MN, USA, 2012, 4328-4333. 
[27] Brown E, Rodenberg N, Amend J, Mozeika A, Steltz E, Zakin M R, Jaeger H M. Universal robotic gripper based on the jamming of granular material. Proceedings of the National Academy of Sciences, 2010, 107(44), 18809-18814.

[28] Kim Y J, Cheng S, Kim S, Lagnemma K. A novel layer jamming mechanism with tunable stiffness capability for minimally invasive surgery. IEEE Transactions on Robotics, 2013, 29 (4), 1031-1042.

[29] Davis S, Tsagarakis N, Canderle J, Caldwell D. Enhanced Modelling and Performance in Braided pneumatic Muscle Actuators. International Journal of Robotics Research, 2003, 22(3), 213-227.

[30] Jiang A, Xynogalas G, Dasgupta P, Althoefer K, Nanayakkara T. Design of a variable stiffness flexible manipulator with composite granular jamming and membrane coupling. Proceedings of the IEEE International Conference on Intelligent Robots and Systems, Vilamoura, Portugal, 2012, 2922-2927.

[31] Godage I S, Branson D T, Guglielmino E, Medrano-Cerda G A, Caldwell D. Shape function-based kinematics and dynamics for variable length continuum robotic arms. Proceedings of the IEEE International Conference on Robotics and Automation, Shanghai, China, 2011, 452-457.

[32] Ziegler J G, Nichols N B. Optimum settings for automatic controllers. trans. ASME, 1942, 64(11).

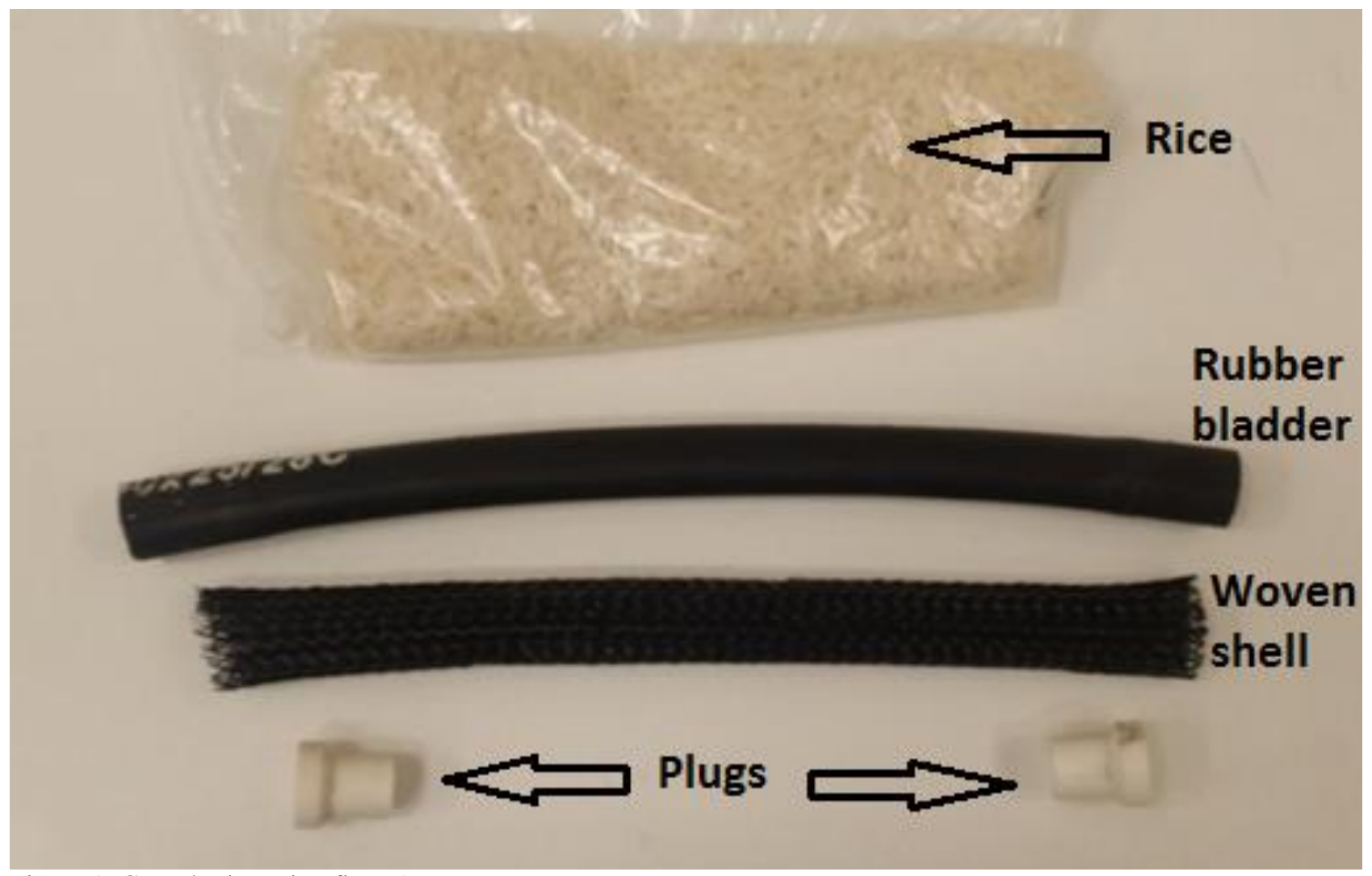

Figure 1: Granular jamming finger's components. 


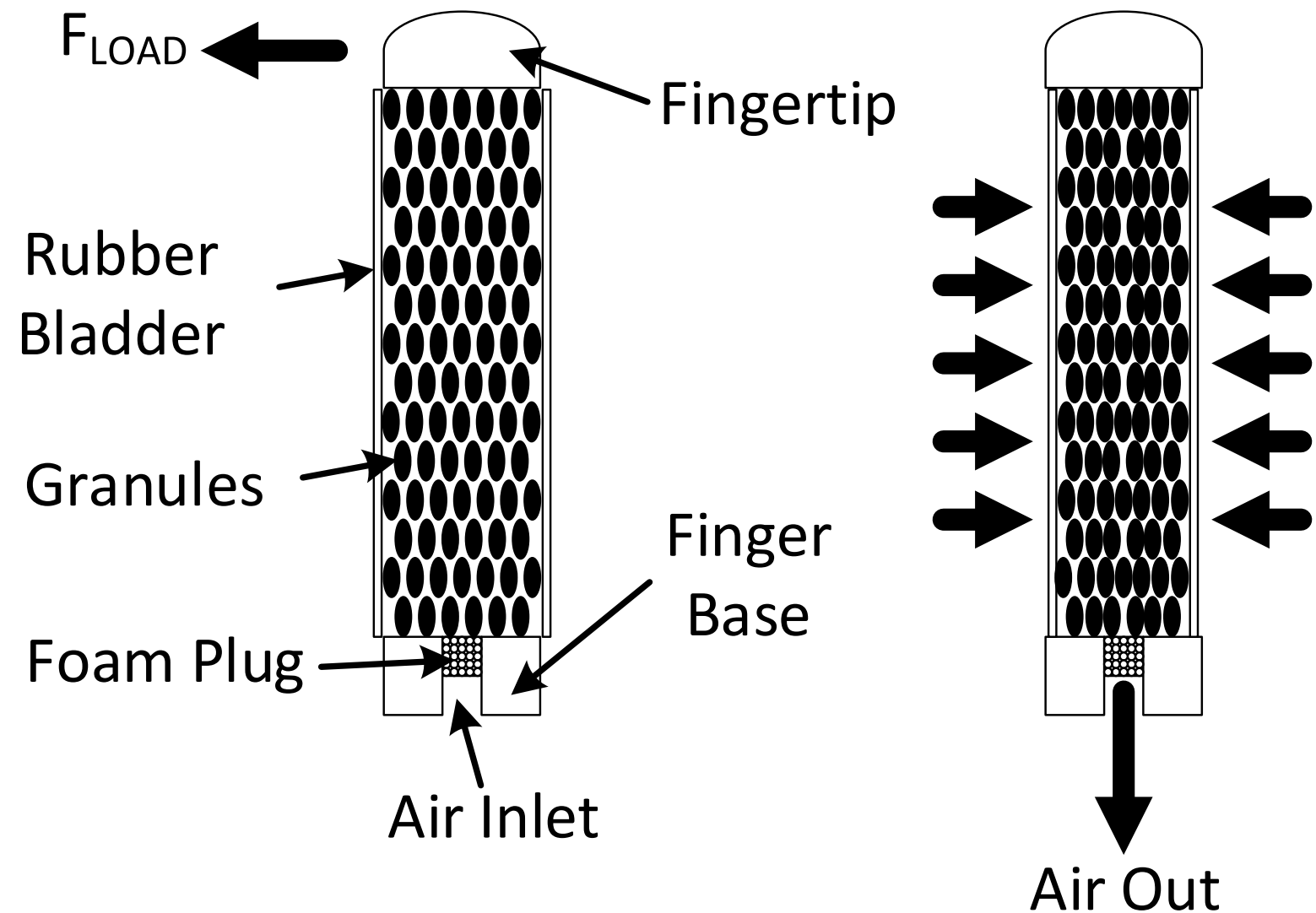

Figure 2: Stiffness changing mechanism in the granular jamming finger.

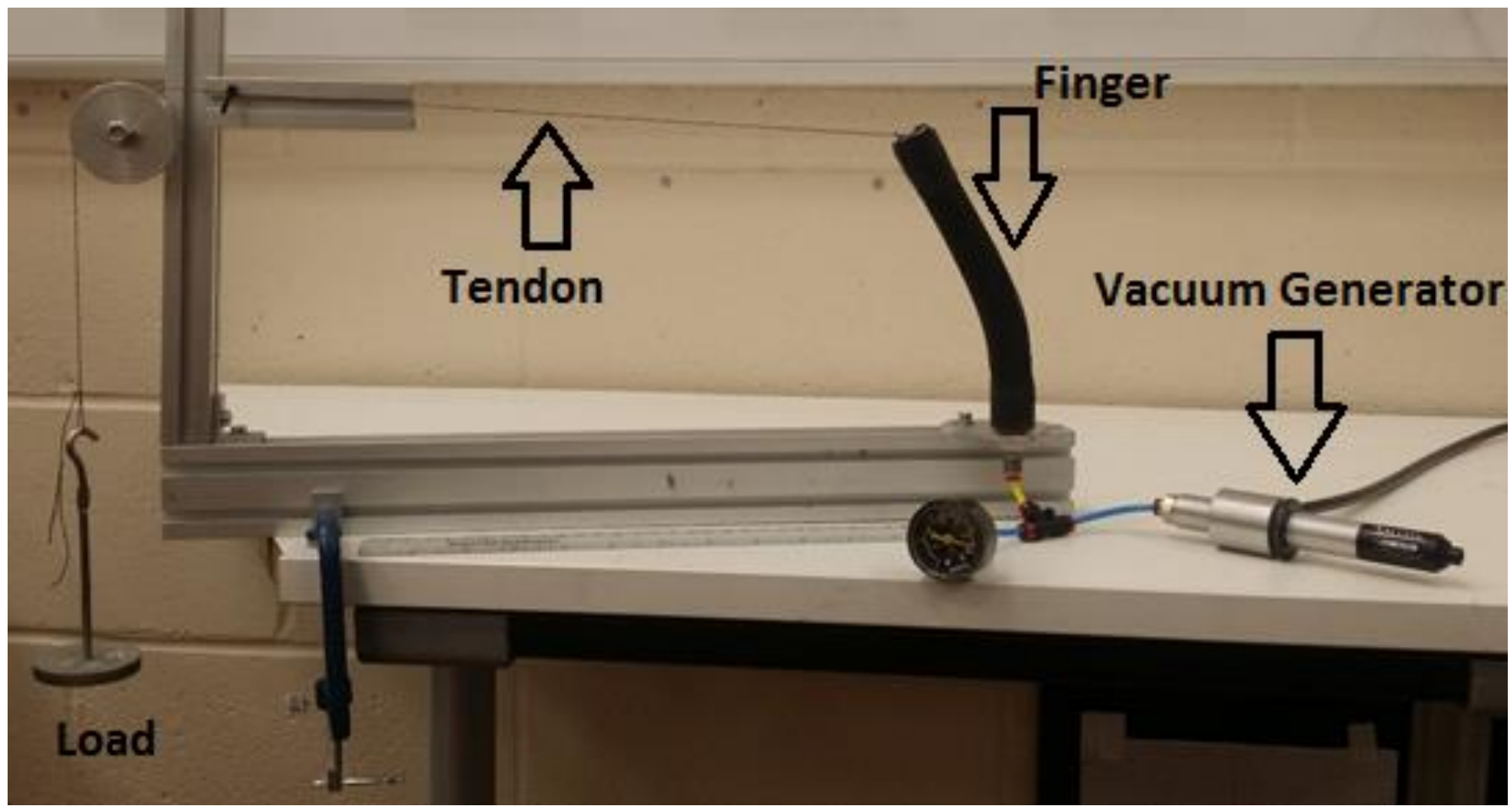

Figure 3: The granular jamming finger test rig. 
A Variable Stiffness Soft Gripper Using Granular Jamming and Biologically Inspired Pneumatic Muscles

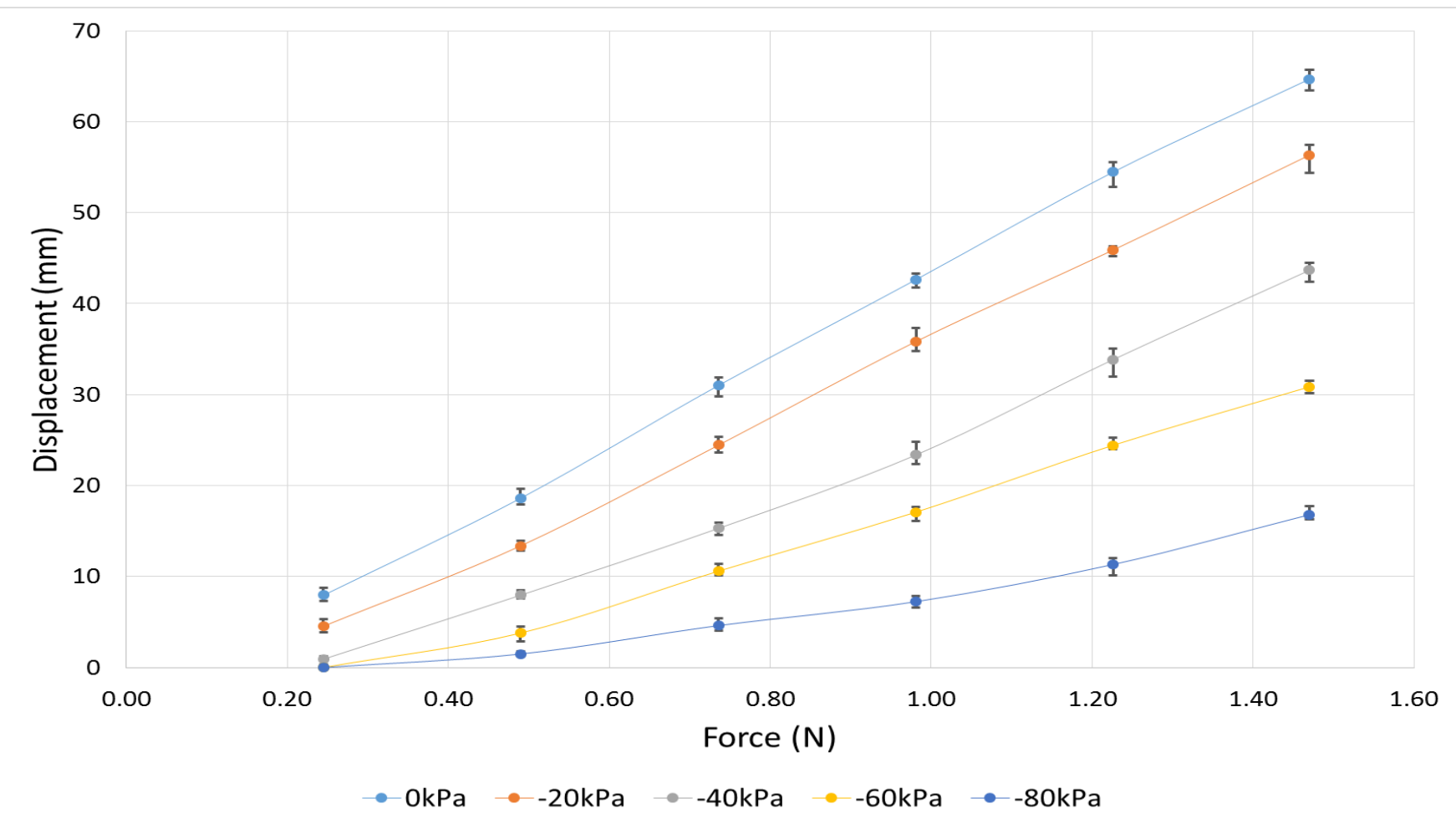

Figure 4: Force/displacement characteristics for the granular jamming continuum actuator.

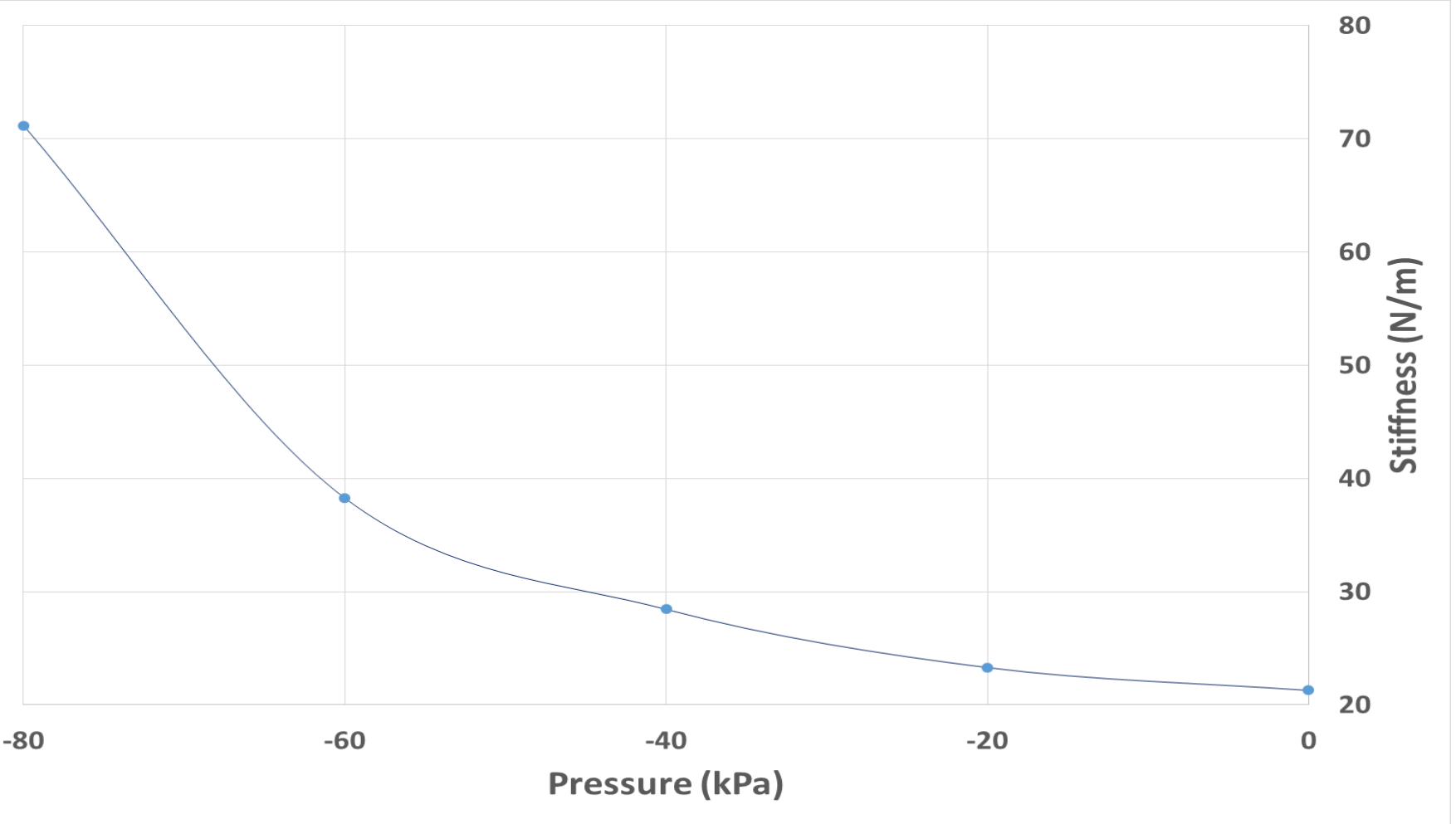

Figure :5 Bending stiffness of the granular jamming continuum actuator. 
A Variable Stiffness Soft Gripper Using Granular Jamming and Biologically Inspired Pneumatic Muscles

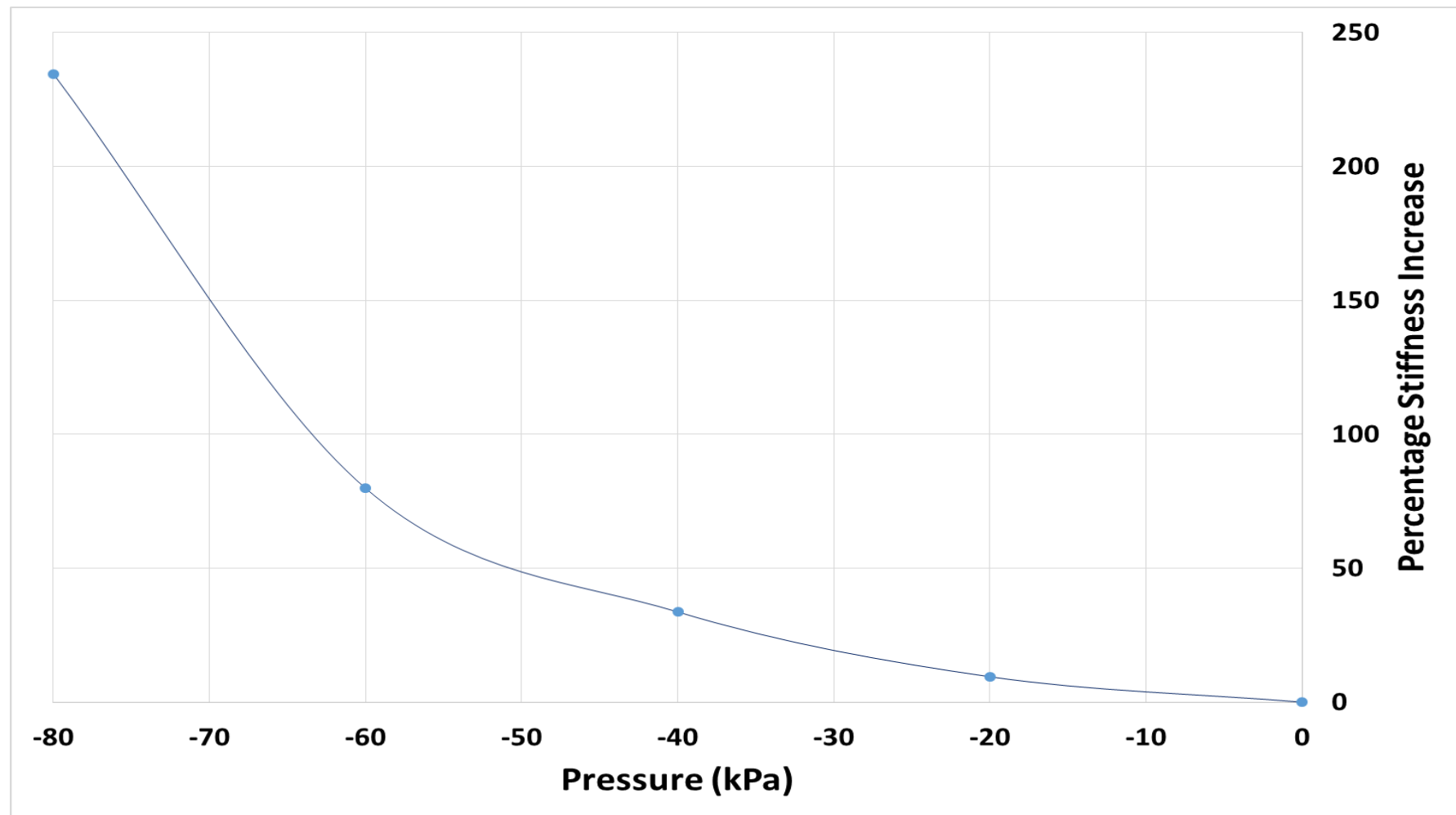

Figure 6: Percentage increase in the granular jamming continuum actuator bending stiffness.

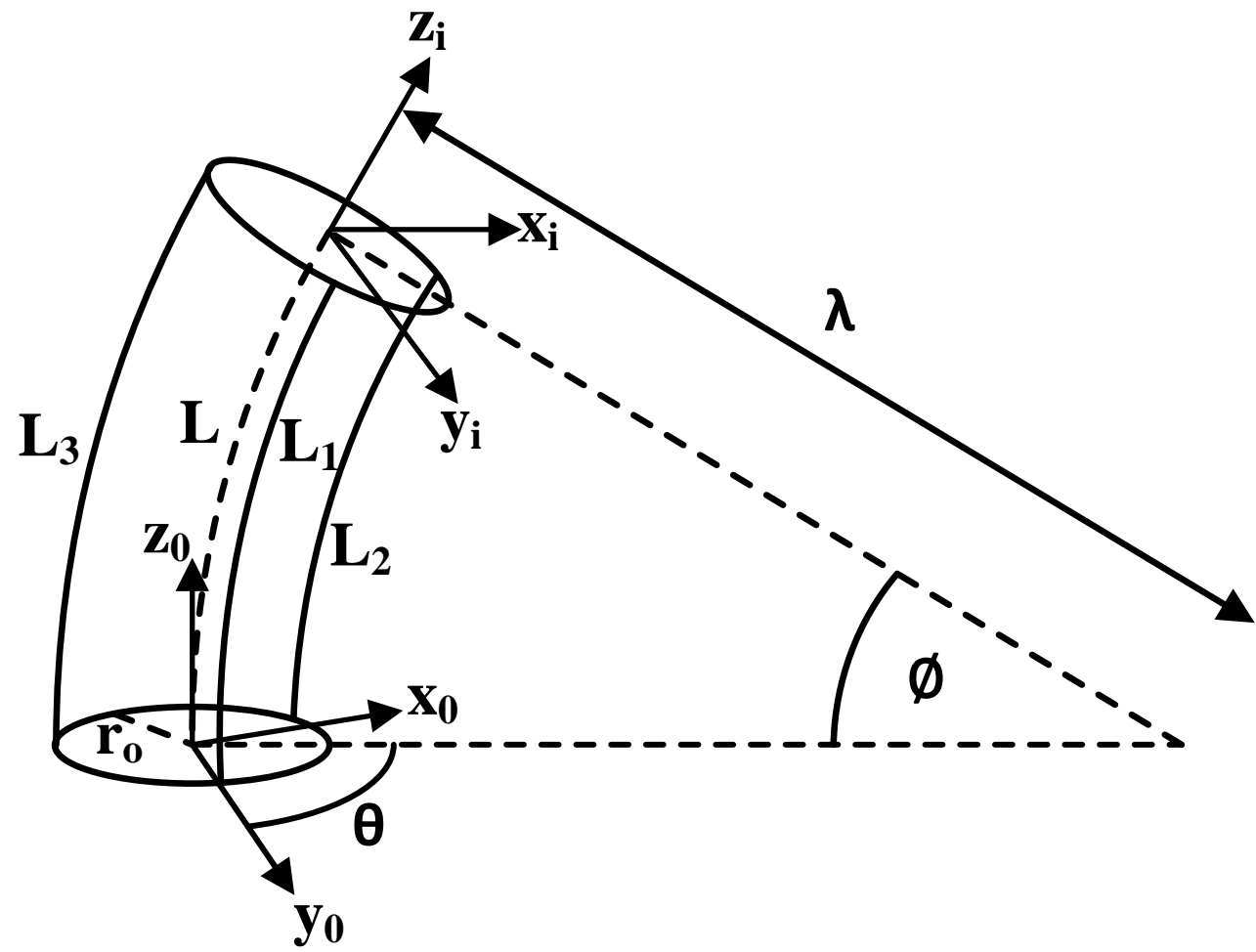

Figure 7: The kinematics for the continuum finger. 


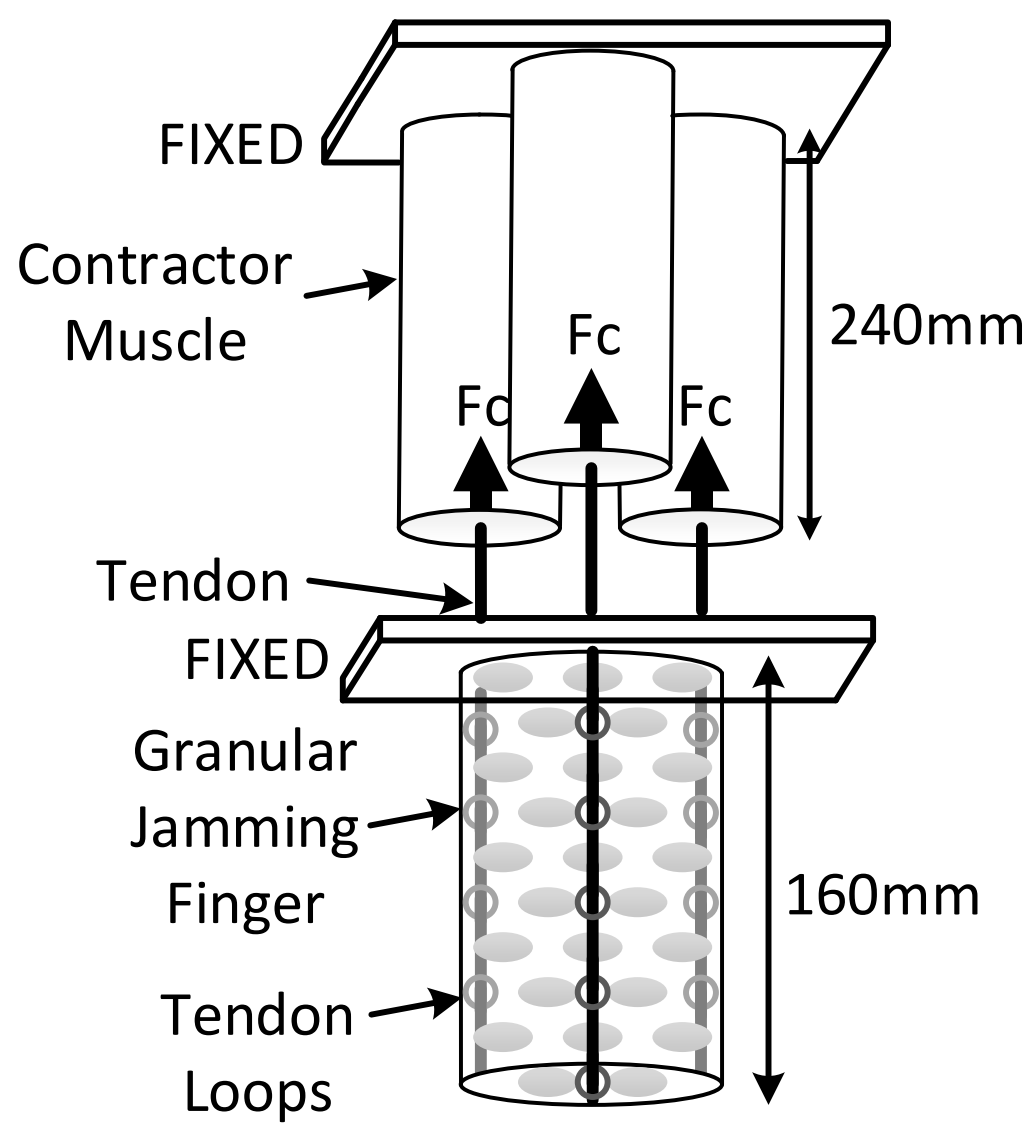

Figure 8: Conceptual design of a single actuated granular jamming finger. 
A Variable Stiffness Soft Gripper Using Granular Jamming and Biologically Inspired Pneumatic Muscles

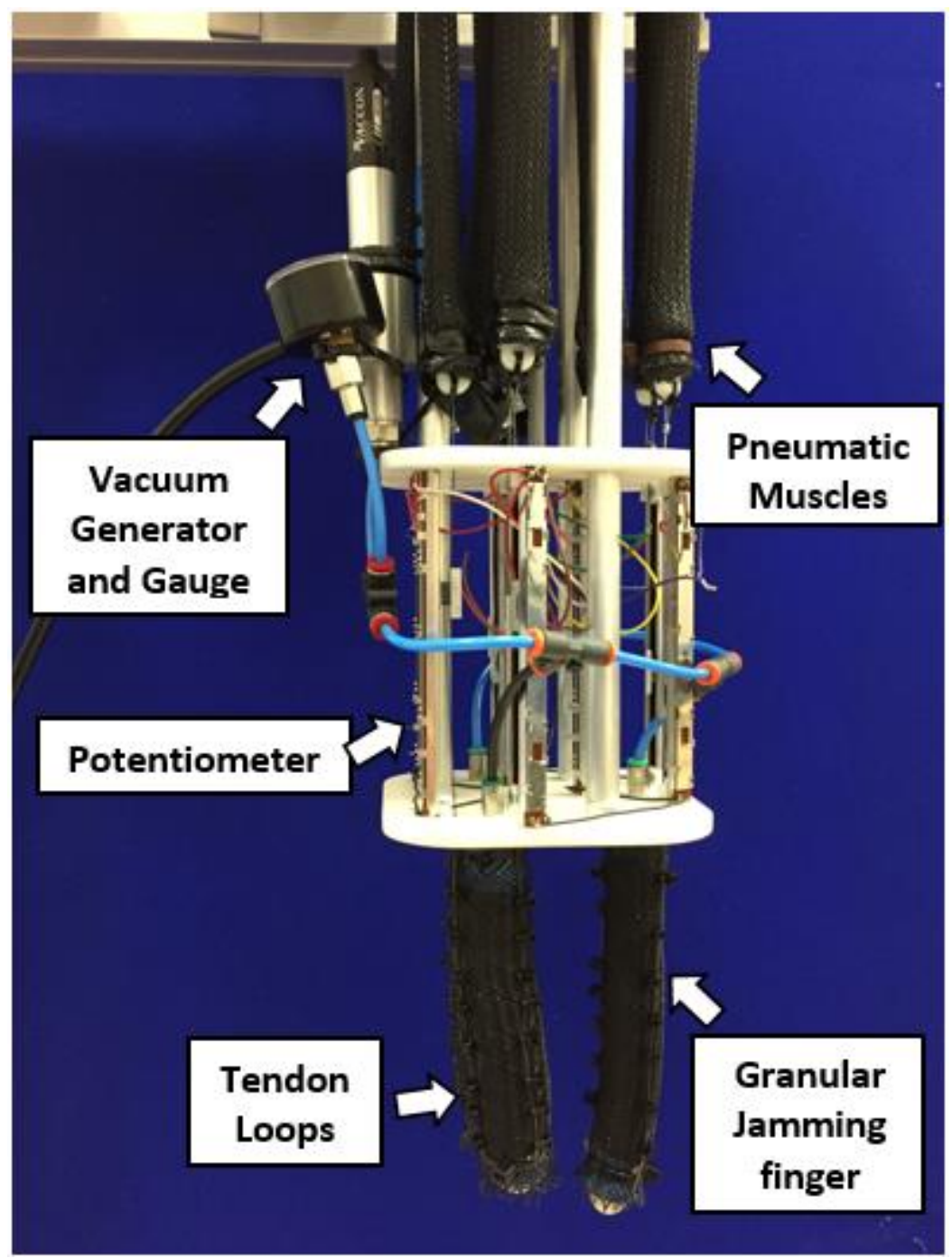

Figure 9 - Variable stiffness granular jamming soft robot end effector. 
A Variable Stiffness Soft Gripper Using Granular Jamming and Biologically Inspired Pneumatic Muscles

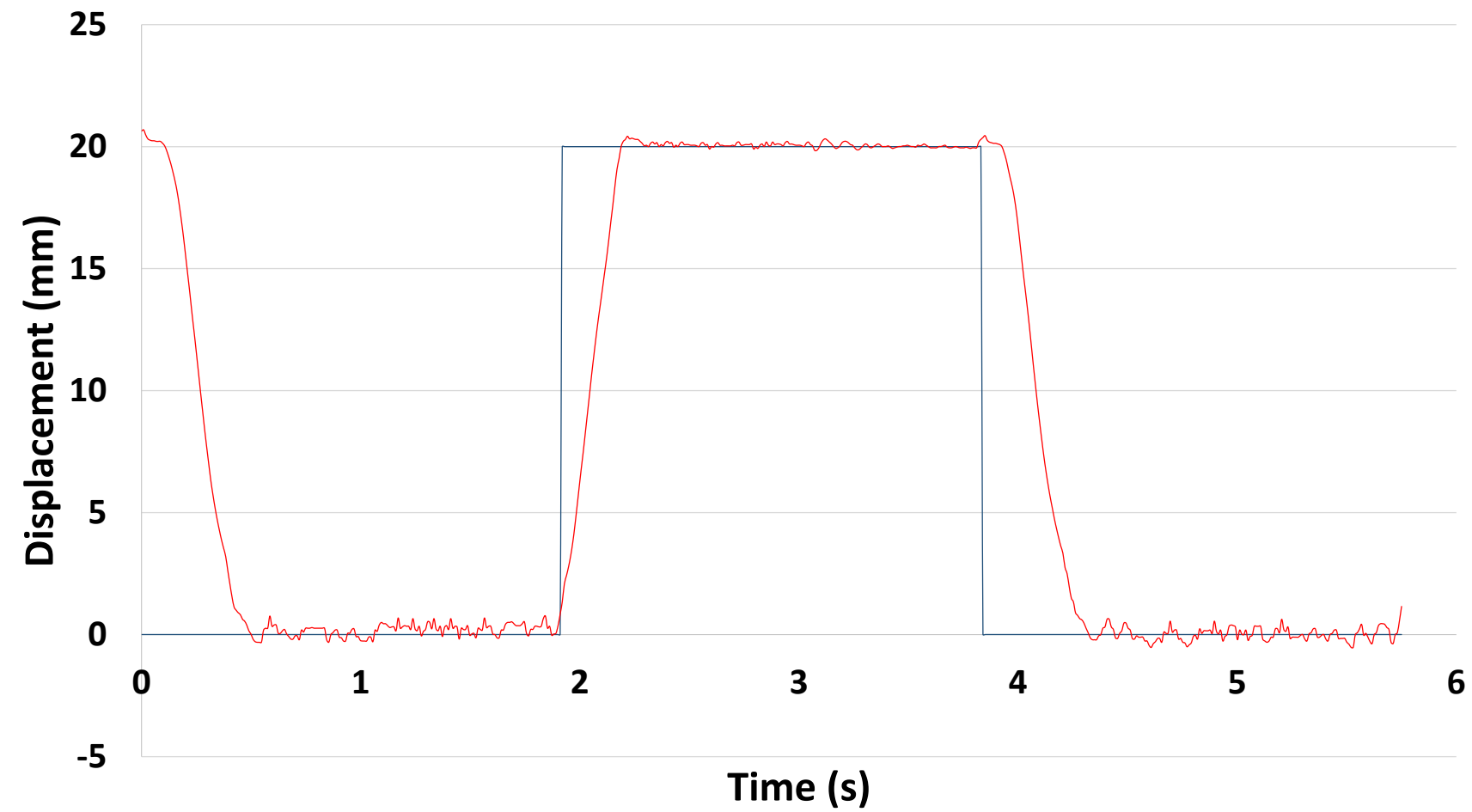

First pneumatic muscle (Finger flexor).

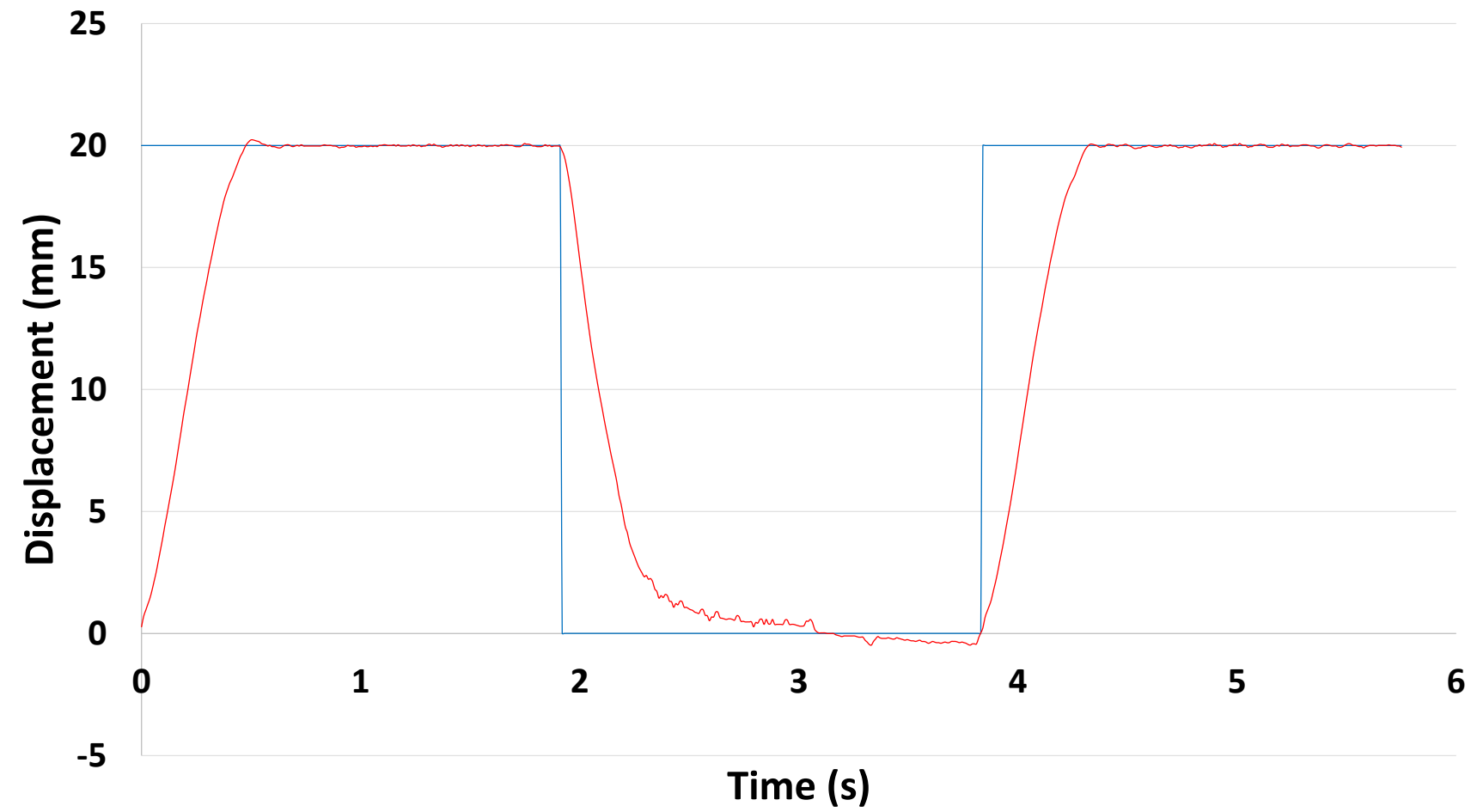

Second pneumatic muscle (finger extensor). 
25

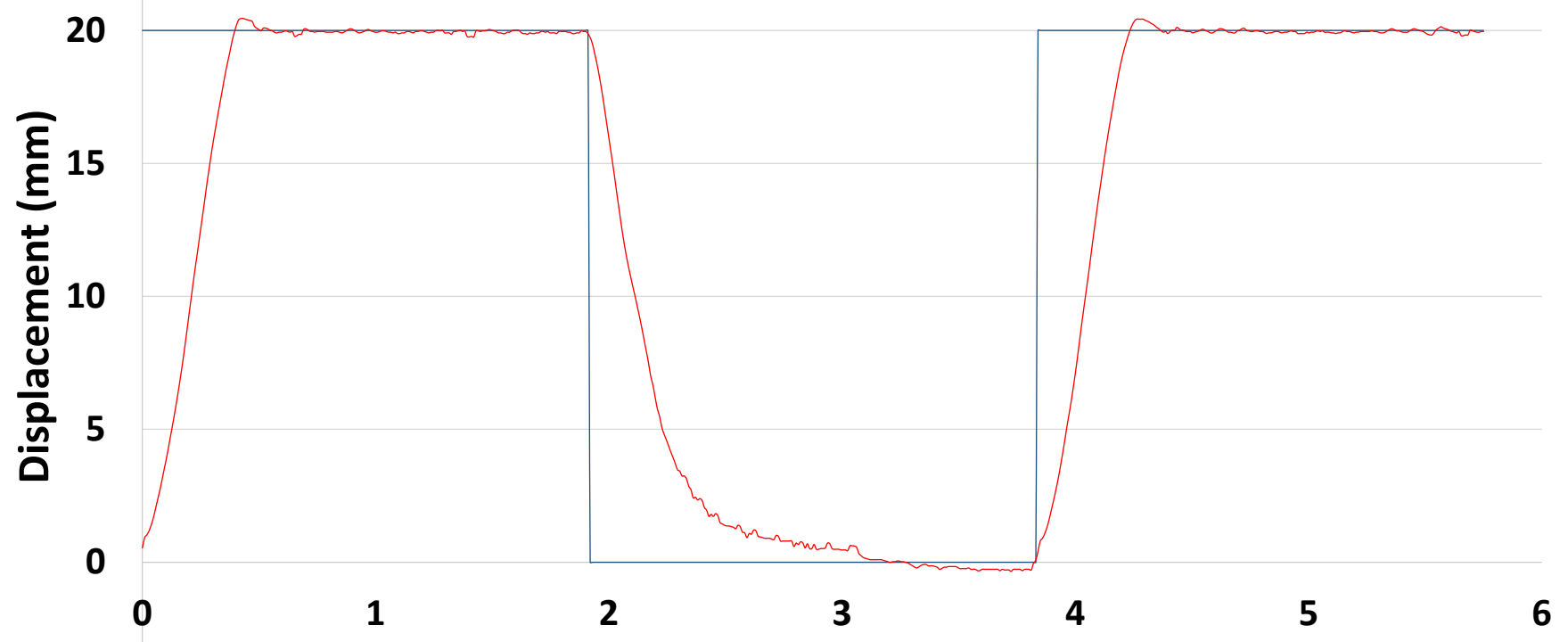

$-5$

\section{Time (s)}

Third pneumatic muscle (finger extensor).

Figure 10: The response of the three contractor muscles when producing a step change in the finger displacement with a 0.0 $\mathrm{kPa}$ pressure. In the experiment bending was in the direction of the first muscle hence the first muscle caused finger flexion and the other two muscles caused finger extension.

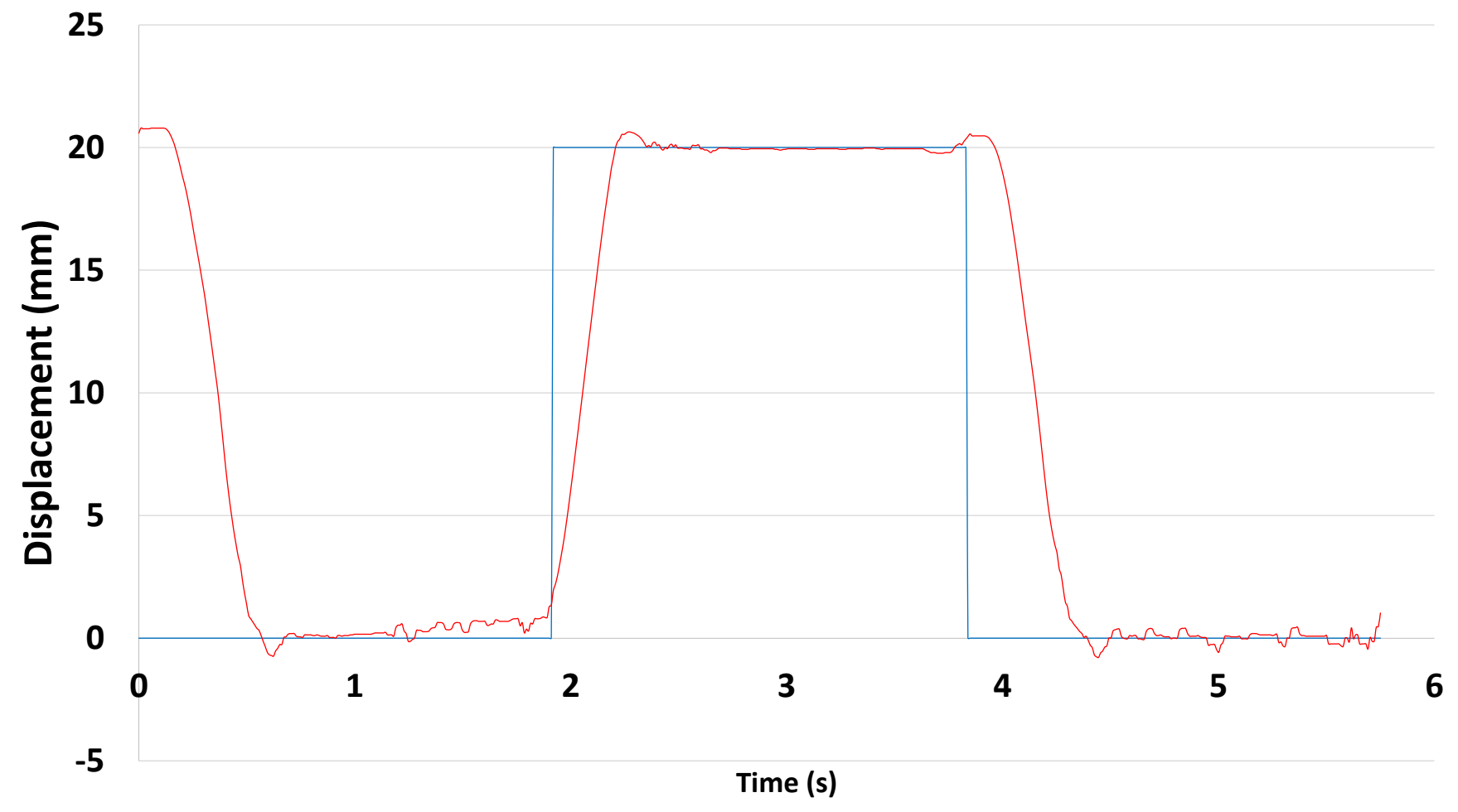

Figure 11: The response of muscle used for finger flexion with a $-40.0 \mathrm{kPa}$ jamming pressure causing a $34 \%$ stiffness increase. 
A Variable Stiffness Soft Gripper Using Granular Jamming and Biologically Inspired Pneumatic Muscles

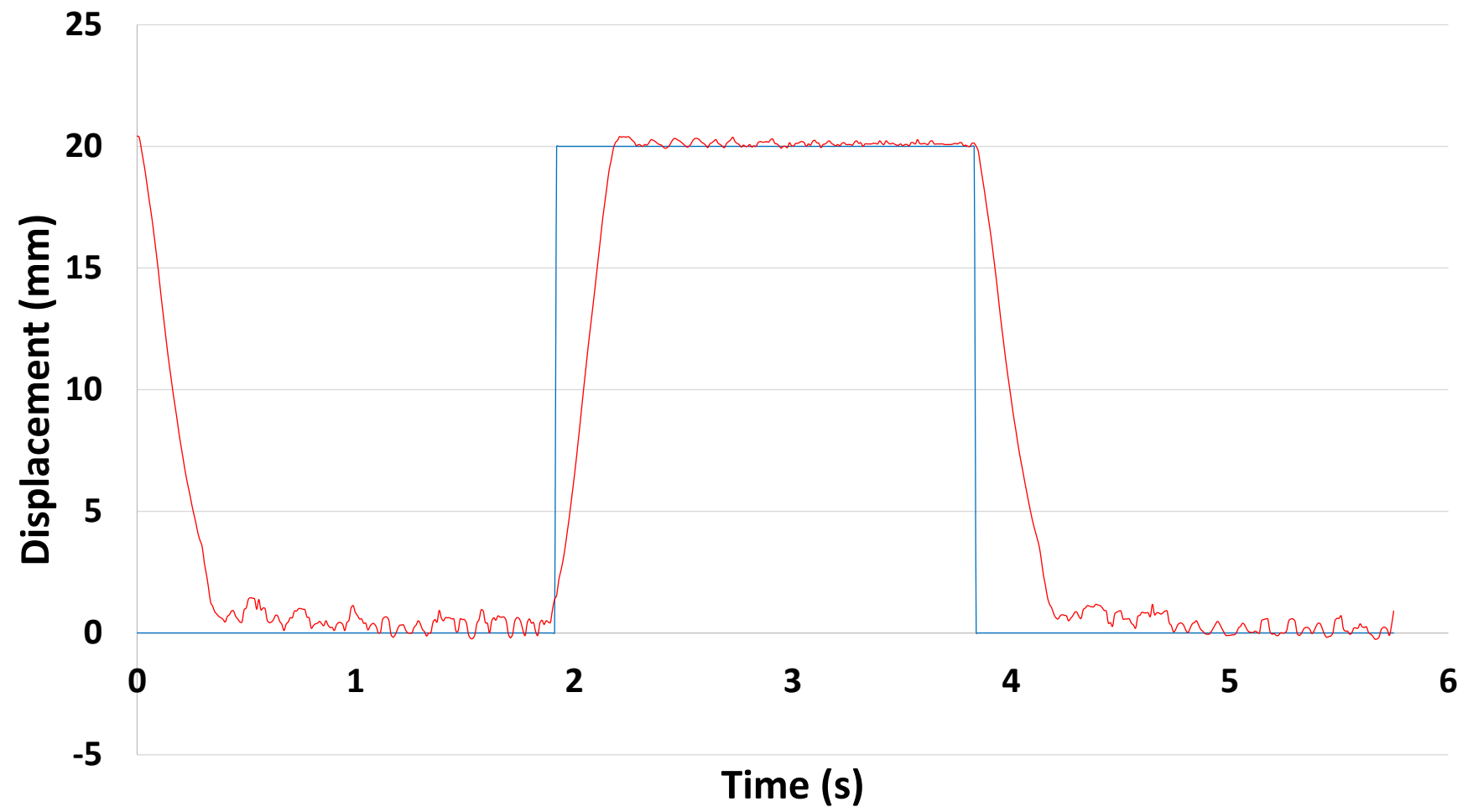

Figure 12: The response of muscle used for finger flexion with a $-80.0 \mathrm{kPa}$ jamming pressure causing a $235 \%$ stiffness increase.

25

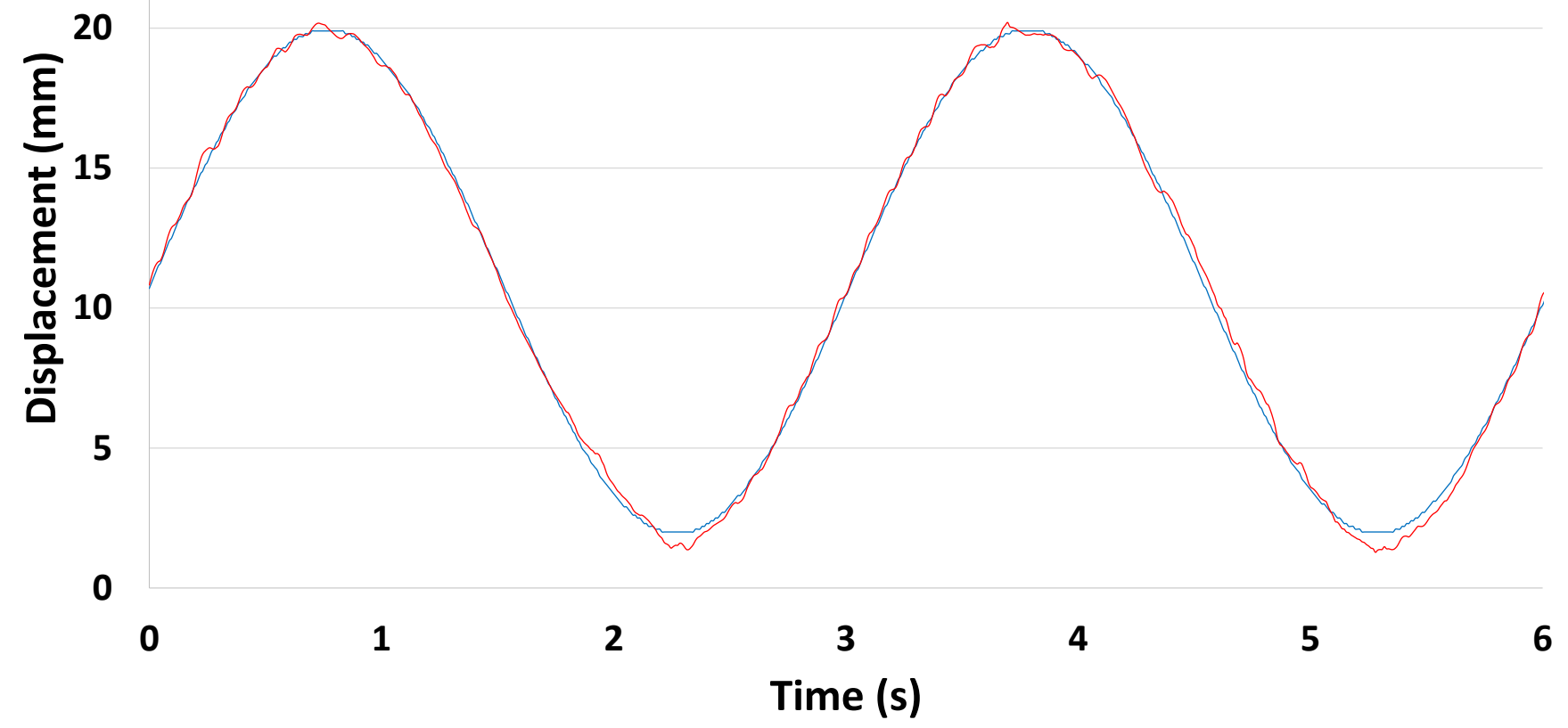

Figure 13: The response of the muscle used for finger flexion tracking a sin wave with a jamming pressure of $0.0 \mathrm{kPa}$. 
A Variable Stiffness Soft Gripper Using Granular Jamming and Biologically Inspired Pneumatic Muscles

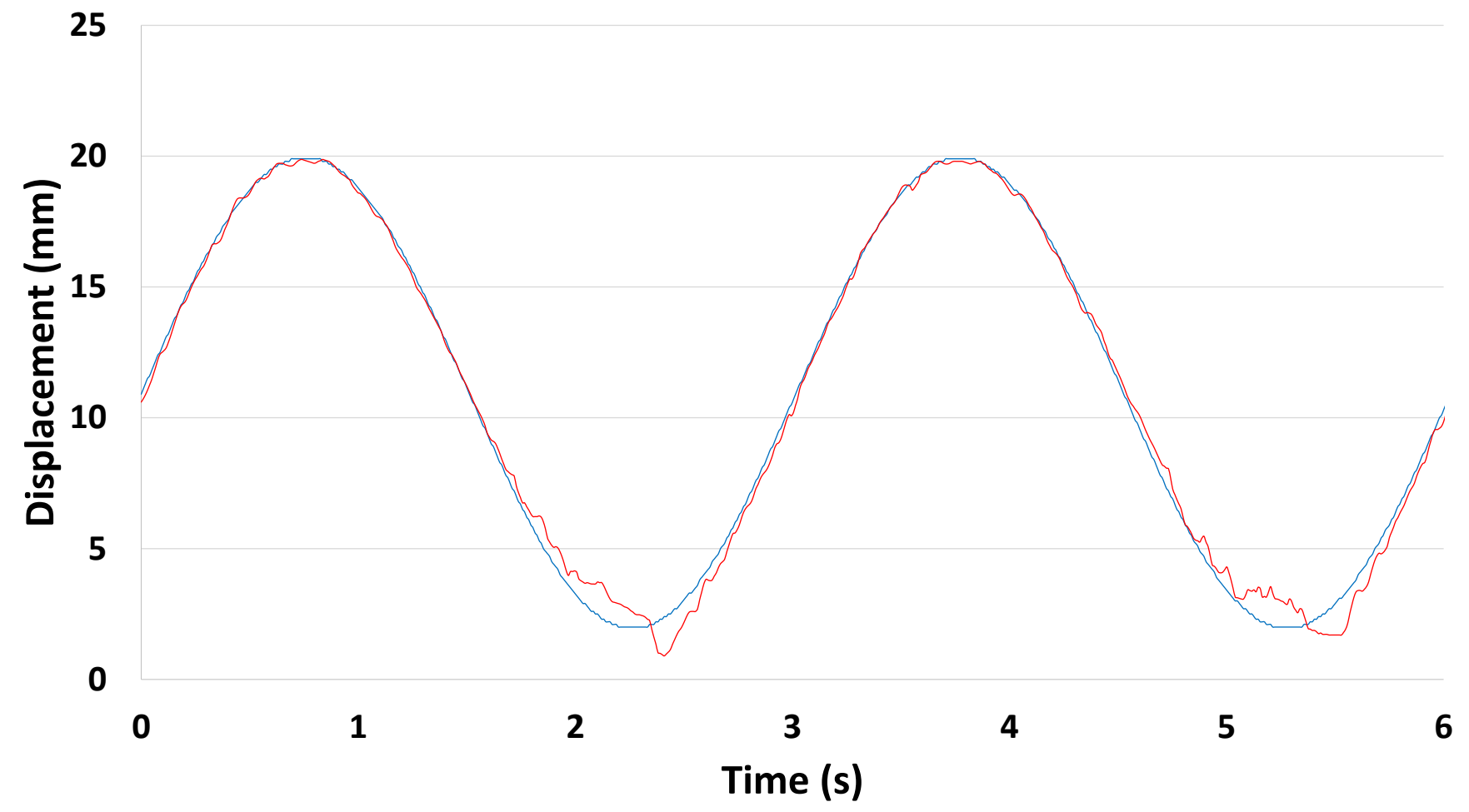

Figure 14: The response of the muscle used for finger flexion tracking a sin wave with a jamming pressure of $-40.0 \mathrm{kPa}$.

25

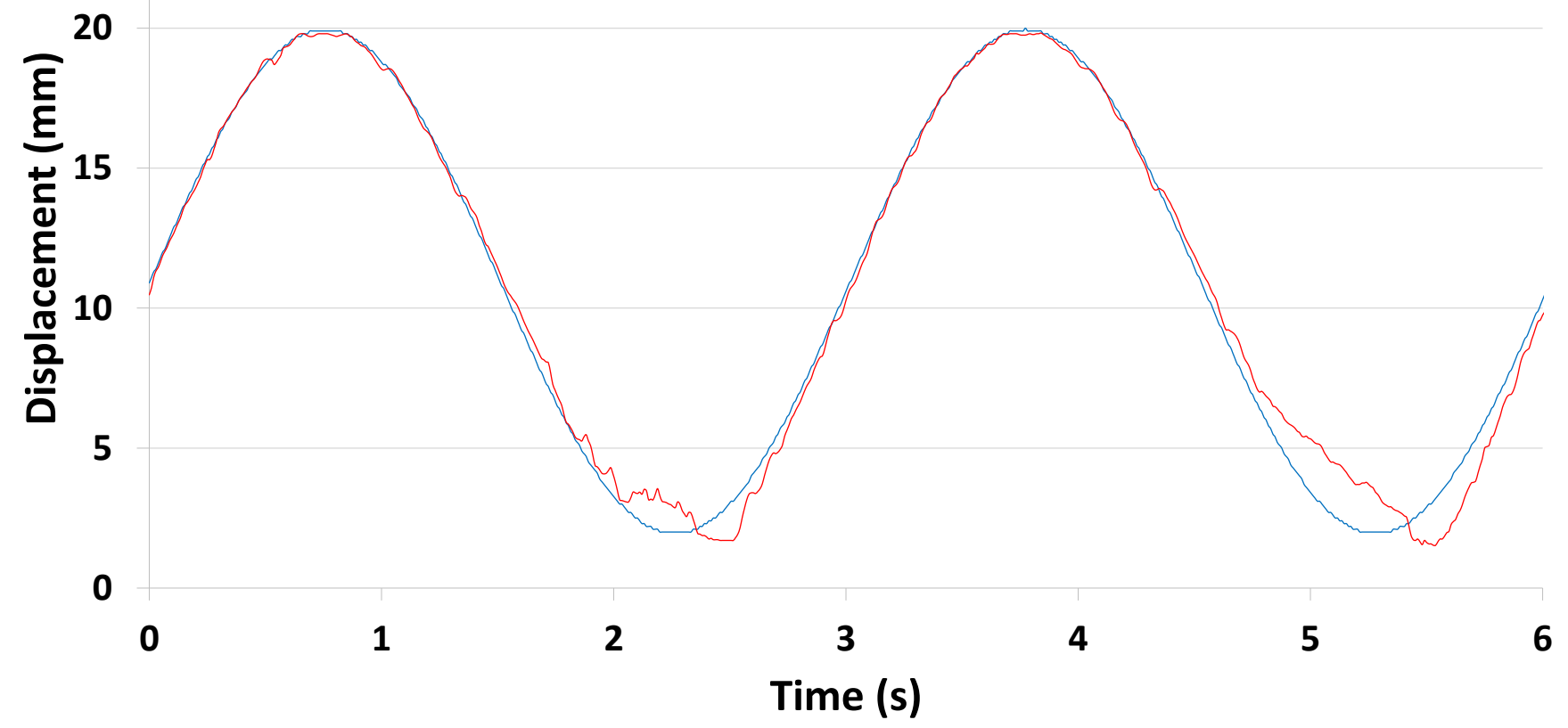

Figure 15: The response of the muscle used for finger flexion tracking a sin wave with a jamming pressure of $-80.0 \mathrm{kP}$ 
A Variable Stiffness Soft Gripper Using Granular Jamming and Biologically Inspired Pneumatic Muscles

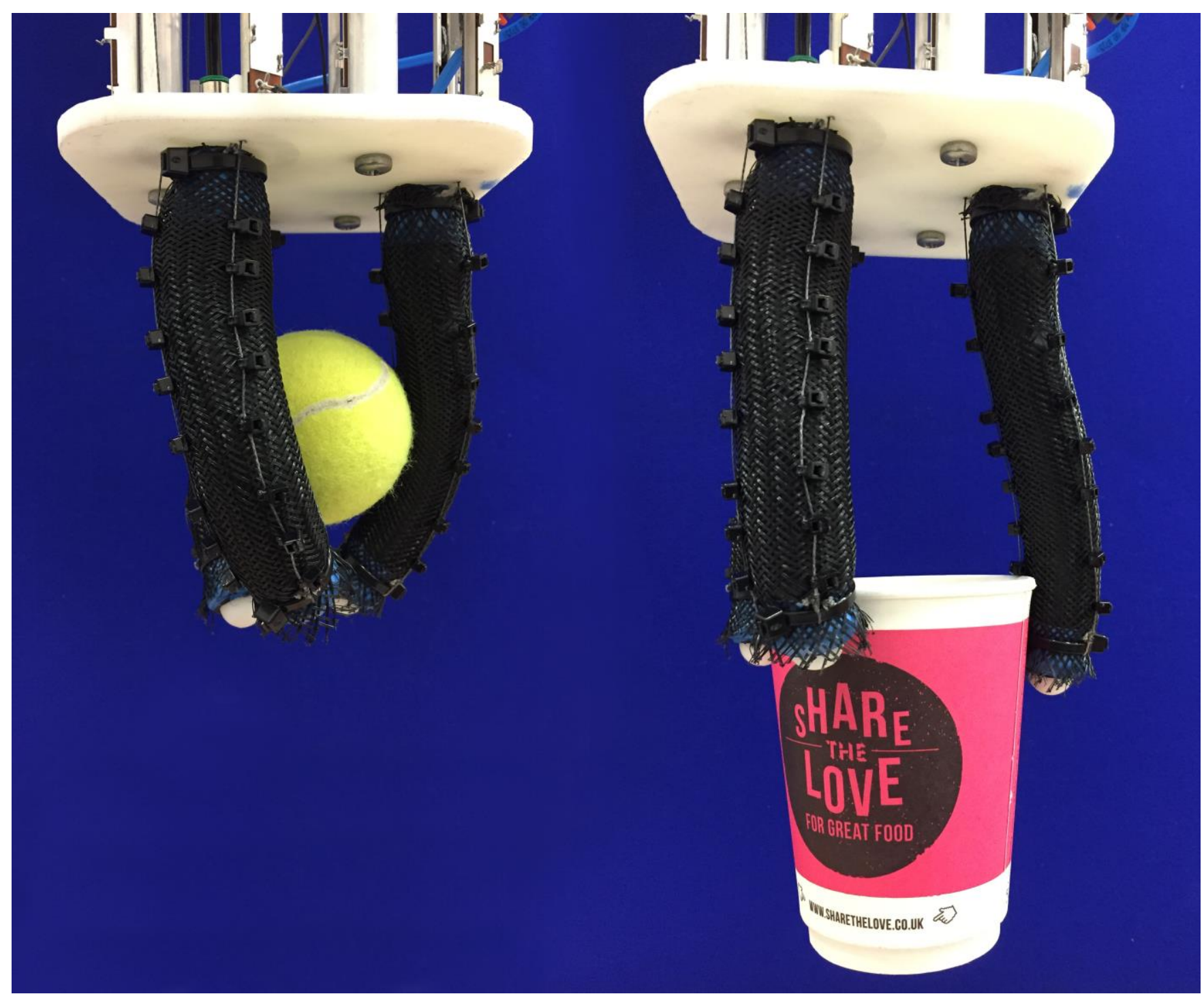

(a)

(b)

Figure 16: Granular jamming grippergrasping objects in different stiffness modes. 